\title{
Objective non-intrusive markers of sperm production and sexual activity
}

\author{
Thilee Sivananthan, Franz Bathur, Mark Jimenez, Ann Conway, Amanda Idan and David Handelsman
}

Objective studies of men's reproductive function are hindered by their reliance on: (i) self-reporting to quantify sexual activity and (ii) masturbation to quantify sperm output rendering both types of estimate vulnerable to unverifiable subjective factors. We therefore examined whether detection of spermatozoa and measurement of prostate-specific antigen (PSA) in urine could provide objective semiquantitative estimates of sperm output and recent ejaculation, respectively, using widely available laboratory techniques. Of 11 healthy volunteers who provided urine samples before and at intervals for 5 days after ejaculation, sperm was present in $2 / 11$ men before, and in all 11/11 samples immediately after ejaculation, but by the second and subsequent void, spermatozoa were present in $\sim 10 \%$. PSA was detectable at high levels in all urine samples, peaking at the first post-ejaculatory sample but returning to baseline levels by the second post-ejaculatory void. We conclude that urinary spermatozoa and PSA are objective biomarkers for sperm production and sexual activity, but only for a short-time window until the first post-ejaculatory urine void. Hence, for a single urine specimen, the presence of spermatozoa and PSA are valid biomarkers, reflecting sperm production and recent ejaculation only until the next micturition, so their measurement should be restricted to the first morning urine void.

Asian Journal of Andrology (2012) 14, 476-480; doi:10.1038/aja.2012.2; published online 23 April 2012

Keywords: ejaculation; PSA; sexual activity; sperm output; spermatozoa; urine

\section{INTRODUCTION}

Major limitations for objective studies of human male reproductive function are the reliance on self-reporting of sexual activity and on masturbation to provide semen samples for analysis of sperm output. Both intrude on sensitive personal matters and rely wholly or partly on subjective aspects of sexual activity. Self-reporting of sexual activity is influenced by complex personal attitudes and perceptions and is effectively impossible to corroborate. Hence, such data are subject to unverifiable bias or distortion. Similarly, the only objective measure of sperm output requires semen specimen collected by masturbation, usually a sensitive and private activity. As a result, men are reluctant to participate in studies requiring semen samples unless actively concerned about their fertility, creating a major participation bias. ${ }^{1-3}$ Hence, the availability of objective, non-intrusive markers of male sexual activity and sperm production could overcome these limitations and, in their absence, these major aspects of male reproductive health remain difficult to study with reliability comparable to many other medical issues.

For example, these limitations make it difficult to estimate accurately the onset of male puberty. Although male puberty usually commences between the ages of 10 and 14 years, the precise timing when sexual activity or sperm production commence is difficult to pinpoint for any individual. While spermarche (the onset of sperm production) can be approximated cross-sectionally in the population and, to a lesser extent, longitudinally in individuals by growth in testicular size, semen analysis is not acceptable for routine clinical evaluation or research investigation of adolescents. Hence, objective estimates of the age of onset of sperm production or sexual activity remain indirect and are of doubtful reliability.

The timing of spermarche was first estimated in 1928 by examining morning urine specimens for spermatozoa ${ }^{4}$ with similar methods developed five decades later by several groups. ${ }^{5-7}$ Although single urine specimens have a relatively high prevalence $(>60 \%)$ of no spermatozoa present even among men with normal spermatogenesis, ${ }^{5-7}$ the cumulative rate of positive samples reaches $80 \%-90 \%$ after five or more daily urine collections. ${ }^{6,8-10}$ The appearance of spermatozoa appears to be random, ${ }^{11}$ although among fertile men having prolonged sexual inactivity, spermaturia is minimal. ${ }^{12}$ This suggests that spermaturia is a regular but intermittent event in sexually active men. This may reflect washout by successive urinations of residual spermatozoa remaining in the urethra after an ejaculation. Assuming this is true, the time window this implies for washout of urethral or bladder spermatozoa has not been characterized.

An adjunct to detection of spermatozoa in urine may be the urinary concentration of prostate-specific antigen (PSA). The PSA concentration in semen is about 10 million times greater than that in serum, ${ }^{13}$ which renders PSA a highly sensitive marker for seminal plasma in studies of condom failure ${ }^{14-19}$ or forensic investigation of sexual assault. $^{20-22}$ So far, studies of urinary PSA have mainly focused on their applications in detection of prostatic pathology. Related observations have indicated that urinary PSA is decreased by castration of males, ${ }^{23}$ increased by testosterone administration to women ${ }^{24}$ and is a 
correlate of neonatal and pubertal male sexual development in boys, ${ }^{25}$ as well as reproductive seasonality in male non-human primates. ${ }^{26}$ However, it has not previously been studied as a non-intrusive marker of recent sexual activity. We propose that urine PSA measurement might be a simple objective semiquantitative method indicating recent ejaculation as assessed by a conventional laboratory technique. If validated, it may assist in circumventing ethical and logistic constraints encountered on semen analysis in the study of certain populations such as adolescents or non-infertile men.

This study therefore aimed at evaluating the presence of spermatozoa in urine and urinary PSA concentration as non-intrusive markers for sperm output and recent ejaculation, respectively, and to study the influences on these measures of time from most recent ejaculation and urinations.

\section{MATERIALS AND METHODS}

The study protocol was approved by the institutional ethics committee and volunteers were required to provide written informed consent prior to participation.

\section{Study 1}

Healthy male volunteers over the age of 18 years were recruited from the local community. Men with a history of chronic medical illness, reproductive disorders or taking regular medication affecting the urogenital tract were excluded.

Pre-ejaculatory urine specimens (PRE) were collected by obtaining the first portion of urine and discarding the remainder. Following collection of semen samples by masturbation in a room adjacent to the laboratory, men voided urine into a second specimen container (the first post-ejaculatory void P1) as soon as possible and before leaving the department. For the first five men, the P1 urine specimens were collected by dividing the sample into five consecutive aliquots of $40-50 \mathrm{ml}$ to determine the partitioning of spermatozoa and PSA excretion in the immediate post-ejaculatory urine (PEU) stream. Collecting the PRE specimens, semen samples and P1 PEU void were completed within $2 \mathrm{~h}$.

All participants collected the entire second and subsequent urine specimens voided over the first $24 \mathrm{~h}$ after semen collection (P2, P3, P4 and P5) and then also their first morning urination for the first 4 days after semen collection (D2, D3, D4 and D5). Urine samples collected at home were refrigerated until delivered to the department as soon as possible. In the laboratory, after thorough mixing a 5-ml aliquot of urine was removed and kept frozen for subsequent PSA measurement. The remainder of the urine samples were refrigerated at $4{ }^{\circ} \mathrm{C}$ and analysed for presence of spermatozoa within 2 days.

\section{Study 2}

Men attending the Clinical Andrology Laboratory, Concord Hospital, Sydney for routine semen analysis (infertility evaluation, elective semen cryostorage) were recruited over a 3-month period during 2010. They provided standard semen laboratory (including time since last ejaculation) and study data, as well as a single spot urine specimen prior to collecting their semen sample. A 5-ml aliquot of urine was kept frozen at $-20{ }^{\circ} \mathrm{C}$ until analysis.

\section{Laboratory methods}

Assessment of spermaturia. Semen analysis was undertaken according to the WHO Semen Manual, 4th edition. ${ }^{27}$ Analysis of urine for presence of spermatozoa was performed by using a modified form of the methodology described by Richardson and Short. ${ }^{5}$

Urine specimens were collected in a 24 -h disposable plastic urine collection bag (Uritainer, Bacto Laboratories, Liverpool, NSW, Australia). After each bag was thoroughly mixed by gentle inversion for $30 \mathrm{~s}$, a 200-ml aliquot was removed and placed in four $50-\mathrm{ml}$ conical tubes (Falcon, Becton Dickinson, NJ, USA) before centrifugation $(1000 \mathrm{~g}, 5 \mathrm{~min})$ in a non-refrigerated IEC Centra MP4 centrifuge (International Equipment Co., Needham Heights, MA, USA). The supernatant in each $50-\mathrm{ml}$ tube was decanted and the sediment vortexed (MT19 Auto Vortex mixer, Chiltern Scientific, London, UK) and then combined into two $10-\mathrm{ml}$ conical tubes. These were then centrifuged again $(1000 \mathrm{~g}, 5 \mathrm{~min})$ with the supernatant again aspirated, leaving $\sim 1 \mathrm{ml}$ urine. The residual urine and sediment were well mixed by agitation before a $10-\mathrm{ml}$ droplet from each tube was analysed for the presence of sperm microscopically at $\times 400$ magnification. Each urine specimen was assessed by a single examiner reviewing a single slide. The number of spermatozoa per slide was assessed and a urine specimen was classified as positive if any spermatozoa were seen either drop.

PSA analysis. PSA was measured by time-resolved fluorescence immunoassay (DELFIA, PerkinElmer, Waltham, MA, USA) in duplicate $20 \mu$ dilutions $(1: 100)$ of urine and the recommended $3-\mathrm{h}$ incubation period. Female urine was used as a blank diluent for assay samples and standards. PSA is stable in serum for up to a week at room temperature. ${ }^{28,29}$

Statistical analysis. The results are expressed as median and range due to the non-normal distribution of variables. Data analysis was performed by Mann-Whitney $U$ test, linear and Spearman correlation, with log transformation of urine PSA concentration, as required using SPSS version 17.0 software (IBM SPSS, Chicago, IL, USA).

\section{RESULTS}

\section{Spermaturia}

In study 1, of the 11 non-azoospermic men, two had PRE specimens positive for spermatozoa. Nine had normal and two had oligozoospermic semen samples. All men completed the PRE sample, semen collection and the P1 PEU voiding within $2 \mathrm{~h}$.

All men had spermatozoa in their first post-ejaculation voiding (Figure 1). In the first five subjects where PEU was collected in five fractions, all five men had spermatozoa in the first fraction, but only three still had spermatozoa present in the fifth fraction. Two men each had spermatozoa present in a subsequent urine specimen collected $24 \mathrm{~h}$ after

Table 1 Study 1: baseline characteristics of men in the study (data are presented as median and interquartile range)

\begin{tabular}{lccccc}
\hline Group & $\mathrm{n}$ & Age (year) & Abstinence (day) & Semen volume (mI) & \multicolumn{1}{c}{$\begin{array}{c}\text { Total spermatozoa } \\
\text { (million per ejaculate) }\end{array}$} \\
\hline Group 1 & 5 & $32(23-36)$ & $2(1.5-3.5)$ & $2.2(1.7-3.4)$ & $149(16-296)$ \\
urine (PRE) & $3.4(2.0-4.4)$ & $3(109-433)$ \\
Total & 6 & $24(23-25)$ & $6(3.5-10.0)$ & $2.6(2.2-4.2)$ & $165(30-382)$ \\
\hline
\end{tabular}

Abbreviation: PRE, pre-ejaculatory urine specimen. 


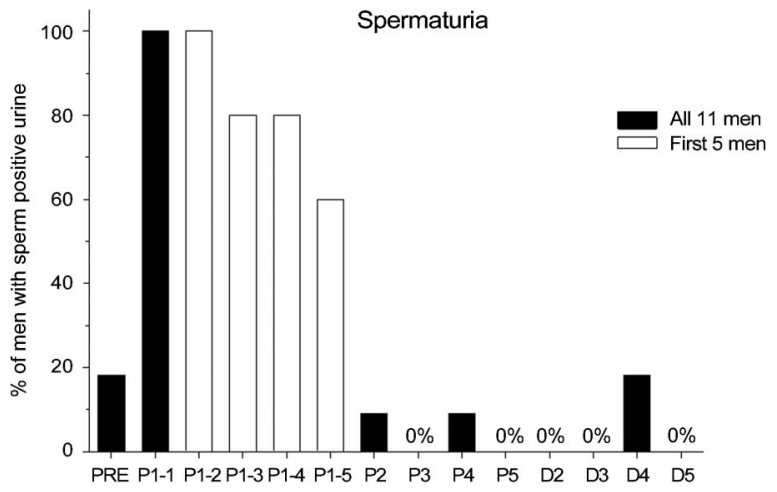

Figure 1 Study 1. Histogram displaying the proportion of 11 men with urine specimens positive for spermatozoa before (PRE) and at intervals after ejaculation. P1 was the first post-ejaculatory void which was further fractionated into five serially-voided aliquots for the first five men only. The subsequent voids P2P5 were within the first day after ejaculation and D2-D5 were the urine samples on the second to fifth day post ejaculation. Further details are shown in Table 1. PRE, pre-ejaculatory urine specimen.

ejaculation. Only two men were positive for spermatozoa in their first morning urine specimens over the remaining 4 days of the study. Both men had ejaculated the evening before the sperm-positive specimen.

\section{Urinary PSA}

In study 1 , urinary PSA concentrations peaked in the P1 specimen, then dropped below the baseline PSA level with the second postejaculatory void (P2), reaching a trough with the next voiding followed by a gradual rise over the next 4 days (D2-D5) (Figure 2). The urinary PSA on day 5 specimens were significantly higher than those in specimens from the second post-ejaculatory void after ejaculation (P2) $(P<0.05)$ (Figure 3).

In study 2, 90 men provided a urine sample before to their semen sample. Of these, six men were omitted from the data analysis (five did not provide a semen sample and one had incomplete data). The remaining 84 men (median age 33.4 years, median abstinence 3.9 days) provided 100 urine samples including 72 men who provided a single urine sample and 12 who provided more than one semen (and urine) sample. All men in study 2 had detectable urinary PSA. There

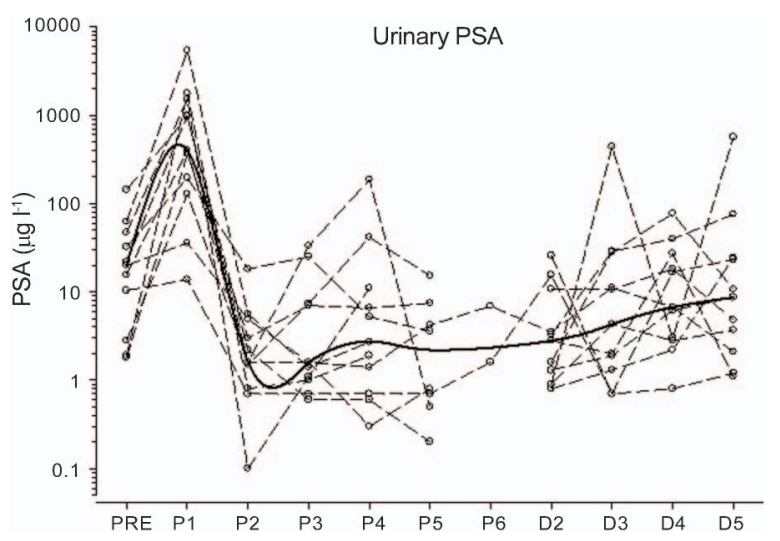

Figure 2 Study 1. Plot of urinary PSA concentration before and at various voids after ejaculation. Note logarithmic $y$ axis scale. Individual men's samples are connected by dotted lines and the solid line is a smoothed spline plot of the median PSA level for each set of void samples. P6 represents 2 men who provided a 6th post-ejaculatory urine sample within the first $24 \mathrm{~h}$ post-ejaculation. Further details are shown in Table 2. PSA, prostate-specific antigen.

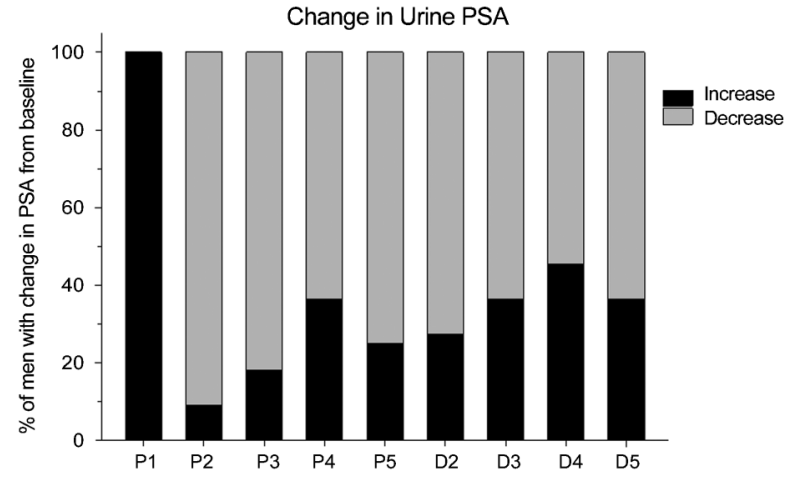

Figure 3 Study 1. Histogram of the proportion of men whose urinary PSA was increased or decreased relative to their pre-ejaculation baseline at various postejaculation time points. PSA, prostate-specific antigen.

was no significant correlation between age or abstinence interval with urinary PSA concentration (Figure 4). Nor was there any statistically significant difference in the age or urinary PSA of men who had abstained for only one day before providing their semen sample and men who had abstained for two or more days.

\section{DISCUSSION}

We were able to demonstrate spermatozoa in the PEU of all 11 men who participated in study 1 . All five men who provided fractionated first void urine samples had spermatozoa in the first post-ejaculatory fraction (P1). Fewer individuals $(3 / 5,60 \%)$ had urine positive for spermatozoa in the last fraction of the first PEU specimen consistent with previous reports. ${ }^{9,30}$ Although both previous studies included fractionated first PEU samples, neither reported data on subsequent voidings after the initial PEU. This study demonstrates that the vast majority of spermatozoa expelled from the urethra by micturition after ejaculation occurs with the first post-ejaculatory voiding.

Only two out of 11 individuals had a urine specimen positive for spermatozoa in a subsequent voiding within the first $24 \mathrm{~h}$. In both, there were only a small number of spermatozoa found in the urine specimens and it is possible that more men may have had some spermatozoa present in their subsequent voiding below the detection limit of the light microscopic detection methodology used. More

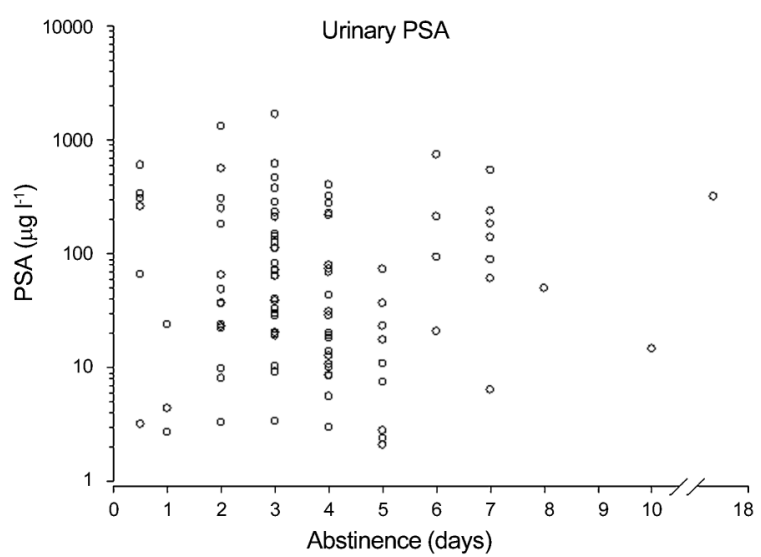

Figure 4 Study 2. Plot of urinary PSA concentration against time since last ejaculation in 100 urine samples from 84 men undergoing routine semen analysis. Note data for 1 day of abstinence include men with less than $24 \mathrm{~h}$ abstinence. PSA, prostate-specific antigen. 
Table 2 Study 2: age and urinary PSA concentration grouped by last reported time since ejaculation

\begin{tabular}{lccccc}
\hline Abstinence (day) & $\mathrm{n}$ & Median age (year) & Age range (year) & Median PSA $\left(\mu g\right.$ I $^{-1}$ ) & ${\text { PSA range }\left(\mu g I^{-1}\right)}^{-1}$ \\
\hline$\leqslant 1$ & 9 & 32 & $18-48$ & 65.8 & $2.7-602$ \\
2 & 15 & 33 & $20-47$ & 37.1 & $3.3-1325$ \\
3 & 30 & 35.5 & $21-48$ & 71.7 & $3.4-1683$ \\
4 & 22 & 33.5 & $18-54$ & 24.5 & $3-405$ \\
$\geqslant 5$ & 24 & 32.5 & $24-56$ & 55.3 & $2.1-1093$ \\
\hline
\end{tabular}

Abbreviation: PSA, prostate-specific antigen.

sensitive methodologies such as flow cytometry would detect more spermatozoa. $^{31}$

Theoretical factors that might determine the presence of spermatozoa in PEU include the total number of sperm cells ejaculated, the number of residual spermatozoa left in the urethra following ejaculation, the time interval, frequency and volume of urine voided since ejaculation, as well as the laboratory methodology used. A longer time interval from ejaculation to the first micturition has been reported to reduce numbers of sperm-positive urine. ${ }^{30}$ Although our study participants provided their first voiding at variable time intervals after ejaculation, all provided their first urine sample within $2 \mathrm{~h}$ of ejaculation. The small number of patients in our study did not permit analysis of the relationship between age, semen volume or total sperm count and a sperm-positive PEU specimen.

In contrast to previous studies ${ }^{9,30}$ reporting absence of spermatozoa in urine before ejaculation in their study subjects, two out of 11 men in our study had spermatozoa in their urine prior to ejaculation with abstinence intervals of 1 and 4 days. Our finding is consistent with a previous report of spermatozoa in the urine of men at up to 9 days of sexual abstinence. ${ }^{12}$ Two other men had sperm-positive morning urinary specimens on day 4 of the study, but both ejaculated again within a day of those urine collections with the sperm-positive urine sample being the first voided after ejaculation. These findings suggest that spermaturia in a first morning urine specimen reflects residual post-ejaculatory sperm in the urethra over a short $(<24 \mathrm{~h})$ time period. This interpretation is consistent with the observation that the prevalence of sperm positivity in random urine specimens is about $30 \%-40 \%$ among men in the reproductive age group who have on average an ejaculatory frequency of 2-3 times per week. ${ }^{32}$ Furthermore, these features also provide an explanation for the observation that the cumulative prevalence of sperm-positive urines increases when collected over consecutive days reaching plateau after 3 days in mature ${ }^{8}$ and 5 days in less mature adolescents. ${ }^{6,8-10}$

Urinary PSA activity was measured in PEU samples from the same urine specimens used to assess spermaturia. To our knowledge, this is the first systematic study of the effect of ejaculation on urinary PSA levels. We observed that in all men urine PSA rises above the baseline pre-ejaculatory level and peaks with the first post-ejaculatory voiding. This rise in urinary excretion of PSA is not sustained beyond the first PEU voiding with all but one man in study 1 registering a decrease in urine PSA level to a level below the baseline level by their second postejaculatory voiding. Hence, the rise in urinary PSA also reflects a short time period. This suggests that, as for spermatozoa, the rise and rapid fall in urinary PSA activity in PEU specimens, reflecting the washout of residual seminal fluid from the urethra, is largely complete with the first PEU voiding.

Previously, the effects of ejaculation on PSA have only been reported in circulating blood. ${ }^{13,33-40}$ In those studies some ${ }^{36,38}$ but not all ${ }^{13,33-35,37,39,40}$ have reported a rise in serum PSA at $24-48 \mathrm{~h}$ after ejaculation. Our findings of a decrease in urine PSA after the first post-ejaculatory voiding is consistent with the timing observed in serum PSA post- ejaculation. ${ }^{13,35,40}$

The transient elevation in post-ejaculatory urinary PSA observed in study 1 was confirmed in study 2 where urinary PSA levels varied widely among men with marked overlap in PSA values for different durations of sexual abstinence (Table 2). Importantly, even men with short periods of abstinence ( 1 day) were found to have urine PSA levels at the lower end of the range. This suggests that the use of random urine PSA to detect recent seminal emission is likely to reflect only very recent ejaculation.

In conclusion, ejaculation results in urinary excretion of spermatozoa in a subsequent urinations, but this appears to be confined largely to the first post-ejaculatory voiding. By light microscopy, reliable detection of spermaturia following an ejaculation or nocturnal emission appears most likely restricted to the first post-ejaculation voiding. The rise and fall of urinary PSA following ejaculation mirrors that of the appearance and disappearance of spermatozoa in urine following ejaculation, both reflecting relatively short-time windows. Hence, detection of spermatozoa or measurement of PSA in urine can reflect recent seminal emission within a relatively narrow interval of time, usually less than a single day.

\section{AUTHOR CONTRIBUTIONS}

TS contributed to the design, statistical analysis and drafting the manuscript and performed most study laboratory procedures. AI recruited and screened volunteers and managed urine samples. FB designed and helped perform the laboratory procedures. MJ validated and performed urine PSA immunoassays. AC contributed to design, monitoring of study and drafting of the manuscript. DH contributed to conception and design and statistical analysis of the study and drafting the manuscript. All authors read and approved the final manuscript.

\section{ACKNOWLEDGMENTS}

T Sivananthan was supported by an Andrology Australia Training Fellowship.

1 Handelsman DJ. Sperm output of healthy men in Australia: magnitude of bias due to self-selected volunteers. Hum Reprod 1997; 12: 2701-5.

2 Cohn BA, Overstreet JW, Fogel RJ, Brazil CK, Baird DD et al. Epidemiologic studies of human semen quality: considerations for study design. Am J Epidemiol 2002; 155: 664-71.

3 Muller A, de la Rochebrochard E, Labbe-Decleves C, Jouannet P, Bujan L et al. Selection bias in semen studies due to self-selection of volunteers. Hum Reprod 2004; 19: 2838-44.

4 Baldwin BT. The determination of sex maturation in boys by a laboratory method. J Comp Psychol 1928; 8: 39-43.

5 Richardson DW, Short RV. Time of onset of sperm production in boys. J Biosoc Sci Supp/ 1978; 10: 15-25.

6 Hirsch M, Shemesh J, Modan M, Lunenfeld B. Emission of spermatozoa: age of onset. Int J Androl 1979; 2: 289-98.

7 Nielsen CT, Skakkebaek NE, Richardson DW, Darling JA, Hunter WM et al. Onset of the release of spermatozoa (spermarche) in boys in relation to age, testicular growth, pubic hair, and height. J Clin Endocrinol Metab 1986; 62: 532-5. 
$8 \mathrm{Ji} \mathrm{CY}$, Ohsawa S. Onset of the release of spermatozoa (spermarche) in Chinese male youth. Am J Hum Biol 2000; 12: 577-87.

9 Weissenberg R, Rozenman H, Hova M, Modan M, Lunenfeld B. The diagnostic value of sperm in post-ejaculatory urine. Int J Androl 1984; 7: 142-8.

10 Schaefer F, Marr J, Seidel C, Tilgen W, Scharer K. Assessment of gonadal maturation by evaluation of spermaturia. Arch Dis Child 1990; 65: 1205-7.

11 Pedersen JL, Nysom K, Jorgensen M, Nielsen CT, Muller J et al. Spermaturia and puberty. Arch Dis Child 1993; 69: 384-7.

12 Barratt CL, Cooke ID. Sperm loss in the urine of sexually rested men. Int J Androl 1988; 11: 201-7.

13 Heidenreich A, Vorreuther R, Neubauer S, Westphal J, Engelmann UH et al. The influence of ejaculation on serum levels of prostate specific antigen. J Urol 1997; 157: 209-11.

14 Walsh TL, Frezieres RG, Nelson AL, Wraxall BG, Clark VA. Evaluation of prostatespecific antigen as a quantifiable indicator of condom failure in clinical trials. Contraception 1999; 60: 289-98.

15 Walsh TL, Frezieres RG, Peacock K, Nelson AL, Clark VA et al. Effectiveness of the male latex condom: combined results for three popular condom brands used as controls in randomized clinical trials. Contraception 2004; 70: 407-13.

16 Chen MP, Macaluso M, Blackwell R, Galvao L, Kulczycki A et al. Self-reported mechanical problems during condom use and semen exposure. Comparison of two randomized trials in the United States of America and Brazil. Sex Transm Dis 2007; 34: 557-62.

17 Macaluso M, Lawson L, Akers R, Valappil T, Hammond K et al. Prostate-specific antigen in vaginal fluid as a biologic marker of condom failure. Contraception 1999; 59: 195-201.

18 Lawson ML, Macaluso M, Duerr A, Hortin G, Hammond KR et al. Partner characteristics, intensity of the intercourse, and semen exposure during use of the female condom. Am J Epidemiol 2003; 157: 282-8.

19 Macaluso M, Lawson ML, Hortin G, Duerr A, Hammond KR et al. Efficacy of the female condom as a barrier to semen during intercourse. Am J Epidemiol 2003; 157: 28997.

20 Hara M, Koyanagi Y, Inoue T, Fukuyama T. [Some physico-chemical characteristics of " -seminoprotein", an antigenic component specific for human seminal plasma. Forensic immunological study of body fluids and secretion.] Nihon Hoigaku Zasshi 1971; 25: 322-4. Japanese.

21 Hochmeister MN, Budowle B, Rudin O, Gehrig C, Borer U et al. Evaluation of prostatespecific antigen (PSA) membrane test assays for the forensic identification of seminal fluid. J Forensic Sci 1999; 44: 1057-60.

22 Sensabaugh GF. Isolation and characterization of a semen-specific protein from human seminal plasma: a potential new marker for semen identification. J Forensic Sci 1978; 23: 106-15.

23 Tremblay J, Frenette G, Tremblay RR, Dupont A, Thabet M et al. Excretion of three major prostatic secretory proteins in the urine of normal men and patients with benign prostatic hypertrophy or prostate cancer. Prostate 1987; 10: 235-43.

24 Obiezu CV, Giltay EJ, Magklara A, Scorilas A, Gooren LJ et al. Serum and urinary prostate-specific antigen and urinary human glandular kallikrein concentrations are significantly increased after testosterone administration in female-to-male transsexuals. Clin Chem 2000; 46: 859-62.

25 Sato I, Yoshikawa A, Fugimoto M, Shimizu K, Ishiwari A et al. Urinary prostate-specific antigen is a noninvasive indicator of sexual development in male children. J Androl 2007; 28: 150-4; discussion 5-7.

26 Sato I, Yoshikawa A, Ishiwari A, Shimizu K. Seasonal changes in urinary prostatespecific antigenic activity in male Japanese macaques (Macaca fuscaa fuscata). J Androl 2007; 28: 821-6.

27 World Health Organisation. WHO Laboratory Manual For The Examination of Human Semen and Sperm-Cervical Mucus Interaction. 4th ed. Cambridge: Cambridge University Press; 1999. 128.

28 Jung K, Lein M, Brux B, Sinha P, Schnorr D et al. Different stability of free and complexed prostate-specific antigen in serum in relation to specimen handling and storage conditions. Clin Chem Lab Med 2000; 38: 1271-5.

29 Woodrum D, French C, Shamel LB. Stability of free prostate-specific antigen in serum samples under a variety of sample collection and sample storage conditions. Urology 1996; 48: 33-9.

30 Engelbertz F, Korda JB, Engelmann U, Rothschild M, Banaschak S. Longevity of spermatozoa in the post-ejaculatory urine of fertile men. Forensic Sci Int 2010; 194: 15-9.

31 Jonckheere J, Callewaert N, Grootenhuis AJ, Kersemaekers WM, Cooper TG. Feasibility study for the centralized measurement of sperm concentration. Int $J$ Androl 2005; 28: 311-5.

32 Smith AM, Rissel CE, Richters J, Grulich AE, de Visser RO. Sex in Australia. Aust NZ J Public Health 2003; 27: 103-256.

33 Glenski WJ, Klee GG, Bergstralh EJ, Oesterling JE. Prostate-specific antigen: establishment of the reference range for the clinically normal prostate gland and the effect of digital rectal examination, ejaculation, and time on serum concentrations. Prostate 1992; 21: 99-110.

34 McAleer JK, Gerson LW, McMahon D, Geller L. Effect of digital rectal examination (and ejaculation) on serum prostate-specific antigen after twenty-four hours. A randomized, prospective study. Urology 1993; 41: 111-2.

35 Simak R, Madersbacher S, Zhang ZF, Maier U. The impact of ejaculation on serum prostate specific antigen. J Urol 1993; 150: 895-7.

36 Tchetgen MB, Song JT, Strawderman M, Jacobsen SJ, Oesterling JE. Ejaculation increases the serum prostate-specific antigen concentration. Urology 1996; 47: $511-6$.

37 Netto NR Jr, Apuzzo F, de Andrade E, Srulzon GB, Cortado PL et al. The effects of ejaculation on serum prostate specific antigen. J Urol 1996; 155: 1329-31.

38 Herschman JD, Smith DS, Catalona WJ. Effect of ejaculation on serum total and free prostate-specific antigen concentrations. Urology 1997; 50: 239-43.

39 Yavascaoglu I, Savci V, Oktay B, Simsek U, Ozyurt M. The effects of ejaculation on serum prostate-specific antigen (PSA). Int Urol Nephrol 1998; 30: 53-8.

40 Stenner J, Holthaus K, Mackenzie SH, Crawford ED. The effect of ejaculation on prostate-specific antigen in a prostate cancer-screening population. Urology 1998; 51: 455-9. 